\title{
Magnetic properties of samples containing small indium particles
}

\author{
J. A. A. J. Perenboom and P. Wyder \\ Physics Laboratory, University of Nijmegen, Nijmegen, The Netherlands \\ F. Meier \\ Laboratorium für Festkörperphysik, Eidgenössiche Technische Hochscule, Zürich, Switzerland
}

(Received 21 July 1980)

\begin{abstract}
Earlier measurements of the magnetization of small indium particles embedded in paraffin were extended in order to observe the transition from a regime of quantum size effects to a regime with normal bulk behavior. Static-magnetization data have been collected in applied magnetic fields up to $8 \mathrm{~T}$ in the temperature range from 3 to $300 \mathrm{~K}$ for samples with a mean particle diameter in the range from 2 to $10 \mathrm{~nm}$. The measured temperature dependence at different values of the applied magnetic field reveals a paramagnetic contribution to the magnetization which can be accurately described with the magnetization of a spin triplet level, $S=1$. The $\mathrm{Cu}$ rie constant is orders of magnitude in excess of one spin per particle and seems to be strongly correlated with the sample handling procedure. In some of our samples we have found also a contribution to the magnetization highly nonlinear with the magnetic field, essentially temperature independent up to room temperature, and saturating at fields around $0.6 \mathrm{~T}$. This contribution resembles strongly the magnetization behavior of ferromagnets. No quantum size effects have been observed in the present data.
\end{abstract}

\section{INTRODUCTION}

During the last four decades there has been a growing interest in the properties of very small metallic particles. In view of the great technological importance of very finely dispersed metals there is an urgent need for a thorough understanding of the character of the transition from the regime of bulk solid behavior to molecular properties. The modifications of the properties of materials resulting from the finiteness of the size of the crystallites deserve careful study.

For several reasons, the magnetic susceptibility of the electrons in very small metallic particles is expected to deviate strongly from normal bulk behavior:

(1) The electrostatic energy required to charge a metallic particle will become larger than the thermal energy at ambient temperature when its size is sufficiently reduced. Because of the resulting improbability of charge fluctuations, the number of electrons contained in a very small metallic particle will be constant. Kubo ${ }^{1}$ was the first to notice this fact and he argued that, consequently, a distinction must be made between particles containing an odd number of electrons ("odd particles") and particles containing an even number of electrons ("even particles"). (2) The spacing between successive energy levels of the electrons in small metallic particles will increase with decreasing particle size. ${ }^{2}$ Deviations from normal bulk solid behavior are believed to occur when the thermal energy $k T$ becomes lower than the average level spacing $\delta$.

Kubo $^{1}$ predicted that the low-temperature susceptibility of an ensemble of small metallic particles will be dominated by the alignment of the one unpaired spin in odd particles: for small enough particles, the Curie-law susceptibility of this single spin will increase over the bulk value of the Pauli spin paramagnetism. The probability distribution of the nearest-neighbor level spacing close to the Fermi energy will strongly influence the theoretical predictions of the detailed temperature dependence of the susceptibility, both for ever and for odd particles. Denton, Mühlschlegel, and Scalapino ${ }^{3}$ have made detailed calculations of the susceptibility and specific heat of the electrons in metal particles in the quantum size regime for different assumptions on the statistics of the level distributions.

Until now the major questions behind the models presented-finite level spacing and even-odd distinction - have not been brought unambiguously to a solution. Knight and co-workers ${ }^{4}$ have observed the deflection of a beam of very small potassium clusters in a magnetic field. The observed intensity profile of the deflected beam suggests the presence of undeflectable even particles and slightly deflected odd particles. More support for the even-odd distinction is found in the nuclear magnetic resonance (NMR) studies on small lithium ${ }^{5}$ and small copper ${ }^{6}$ particles. The NMR resonance was observed to move to lower values of the Knight shift with decreasing temperature and particle size, in accordance with a vanishing 
electronic susceptibility for even particles. The electronic susceptibility of the odd particles is reflected in a low field tail of the NMR signal. The total susceptibility is mainly due to the odd particles and can be determined from the intensity of the conductionelectron-spin-resonance (CESR) signal. In most metals CESR cannot be observed in the bulk due to the fast relaxation, but in very small particles the spin relaxation processes at a resonance frequency $\omega_{c}$ will be quenched with a factor $\hbar \omega_{c} / \delta$ (Ref. 7) when the average level spacing becomes appreciable. In this way CESR has been observed in small silver particles ${ }^{8}$ and the intensity was found to obey a Curie law. In addition, a Curie-law susceptibility of a magnitude of order one spin per particle has been observed in the static susceptibility of small platinum particles with an average diameter of $2 \mathrm{~nm} .^{9}$ The CESR observed in these samples reflected a Curie law, probably due to conduction-electron spin resonance in the small particles, but corresponding to much less than 0.5 spin per particle. ${ }^{10}$

Meier and Wyder ${ }^{11}$ have measured the static magnetization of samples consisting of gas-evaporated indium particles embedded in paraffin. In the temperature interval spanned by the temperature of the liquid helium, from 1.5 to $4.2 \mathrm{~K}$, the magnetization was only weakly temperature dependent (as compared to a $1 / T$ law). A paramagnetic contribution to the magnetization was found in this temperature range, which saturated at fields around $2 \mathrm{~T}$, and which was superposed on a contribution linear with the magnetic field $B$. It was suggested that the relatively low saturation field of the observed paramagnetic magnetization is due to the large orbital momenta associated with the states of the electrons which are confined to the nearly spherical volume of the metal particles. In a more elaborate discussion, van Gelder ${ }^{12}$ was able to modify this model to include the observed temperature dependence. It is obvious that for a reliable assignment of the observed effects to quantum size effects in the small indium particles measurements are needed over a more extended temperature range. Also, the correlation must be verified of the observed effects with the size of the small particles. In this paper we will discuss the results of the extension of the earlier measurements to the temperature range from 3 to $300 \mathrm{~K}$ and in even higher magnetic fields, up to $8 \mathrm{~T}$. It will be shown that the temperature dependence of the magnetization observed over the whole range of temperature and magnetic field is accurately described by a common Langevin function for spin $S=1$. The paramagnetic susceptibility is for all samples of the same order of magnitude as the residual paramagnetic susceptibility observed in blank paraffin samples, and is orders of magnitude higher than expected from quantum size effect predictions. We conclude that no quantum size effects were observed in small indium particles embedded in paraffin.

\section{EXPERIMENTAL DETAILS}

\section{A. Preparation and characterization of the samples}

The small particles have been prepared by evaporation of indium in a background of helium gas. A stream of pure helium gas ${ }^{13}$ is drawn, at a pressure of a few Torr, through a temperature stabilized oven which has been made of quartz. Indium metal ${ }^{13}$ is evaporated from a crucible into the helium gas. The indium particles which are formed are carried as an aerosol to a filter of molten paraffin. The paraffin ${ }^{13}$ is kept at a temperature of $45^{\circ} \mathrm{C}$, slightly above its solidification point, by means of a temperature regulated water supply. With the present setup the indium concentration in the paraffin increases by approximately $1000 \mathrm{ppm} /$ day.

For the determination of the size distribution a small amount of the indium-paraffin suspension was solved in petroleum ether. This solution was treated with ultrasonics to ensure thorough mixing of the colloid. The size distribution was determined from electron micrographs taken from deposits of this dilute solution. The average diameter $d_{\mathrm{av}}$ and the width of the size distribution $\sigma$ have been determined from a best fit of a log-normal size-distribution function ${ }^{14}$ to the size-distribution histogram. The indium concentration, the size-distribution parameters, and the total number $N_{\text {sample }}$ of clusters present in the samples are listed in Table $\mathrm{I}$.

\section{B. Measurement technique}

The magnetic moment of the samples has been determined with a very sensitive magnetometer containing a set of superconducting pickup coils and a current detector in the liquid-helium bath. ${ }^{15}$ The position of the sample was modulated at a very low frequency; the sample moved inside a continuous flow cryostat inserted through the pickup coils. Here, we will give only a brief description of the principles and performance of the present instrument; a full discussion will be presented elsewhere. ${ }^{16}$

The magnetic field of up to $8 \mathrm{~T}$ is generated by a superconductive solenoid. Two oppositely wound pickup coils are positioned coaxially with the magnetic field and they are rigidly attached to the magnet as shown in Fig. 1. In a conventional vibrating sample magnetometer, the induction voltage generated in a suitable set of pickup coils is proportional to the induced magnetic moment of the vibrating sample. ${ }^{17,18}$ In our setup the pickup coils are part of a lowinductance superconducting loop, the flux transformer. Because the flux contained by a closed superconducting circuit is constant, a shielding current will be generated in the flux transformer proportional to the net change in flux sensed by the pickup coils. For 
TABLE I. Experimental results for the samples of indium particles embedded in paraffin and for the blank paraffin samples.

\begin{tabular}{|c|c|c|c|c|c|c|}
\hline & $\begin{array}{c}\chi_{m} T(S=1) \\
\left(10^{-9} \mathrm{~m}^{3} \mathrm{~K} / \mathrm{kg}\right)\end{array}$ & $\begin{array}{c}m_{\text {sat }} \\
\text { ferromagnetic } \\
\left(10^{-9} \mathrm{~J} / \mathrm{T}\right)\end{array}$ & $\begin{array}{c}\text { Indium } \\
\text { concentration } \\
(\mathrm{ppm})\end{array}$ & $d_{\mathrm{av}}(\mathrm{nm})$ & $\sigma$ & $N_{\text {sample }}$ \\
\hline In 1 & $?$ & 120 & 500 & 9.1 & 2.1 & $3.9 \times 10^{11}$ \\
\hline In 2 & Meissner effect & $<20$ & 3000 & & & \\
\hline In 3 & 6 & $<20$ & 1060 & 3.6 & 1.4 & $1.2 \times 10^{14}$ \\
\hline In 4 & 7.3 & $<20$ & 410 & 1.8 & 1.6 & $3.2 \times 10^{14}$ \\
\hline In 5 & 5.5 & $<20$ & 250 & & & \\
\hline In 6 & 11.4 & 200 & 730 & & & \\
\hline In 7 & 16.7 & 210 & 1800 & 4.3 & 2.2 & $7.9 \times 10^{12}$ \\
\hline In 8 & 4.4 & 520 & 1800 & 4.3 & 2.2 & $1.4 \times 10^{13}$ \\
\hline B| 1 & 18 & 1290 & $\cdots$ & & & \\
\hline BI 2 & 9 & $<20$ & $\cdots$ & & & \\
\hline B| 3 & 7.2 & $<20$ & $\cdots$ & & & \\
\hline B| 4 & 18.6 & $<20$ & $\cdots$ & & & \\
\hline B| 5 & 7.2 & $<20$ & $\cdots$ & & & \\
\hline
\end{tabular}

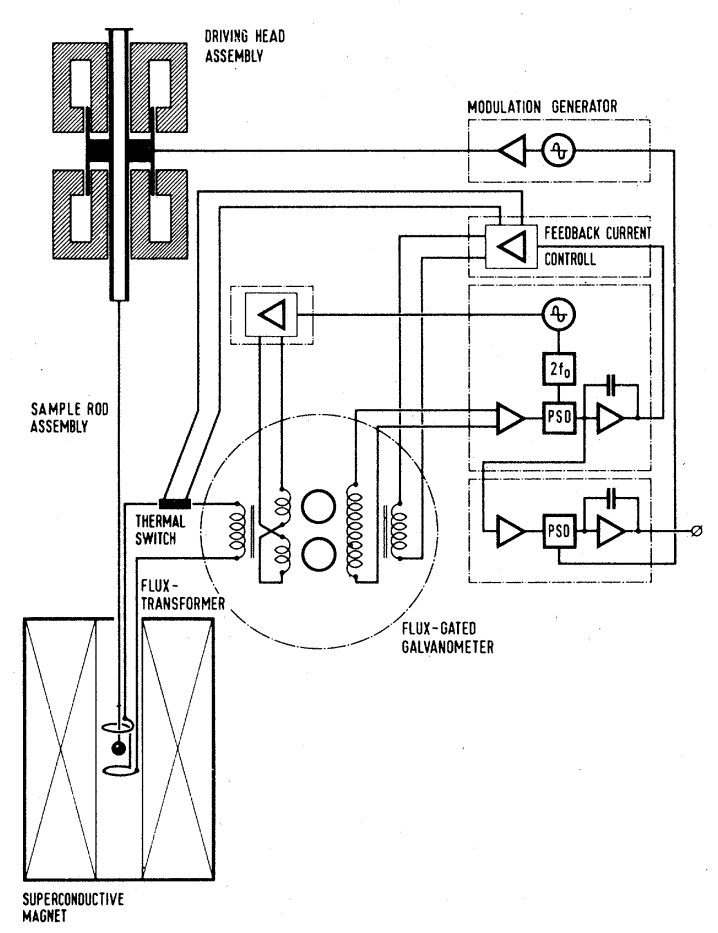

FIG. 1. Schematic diagram of the equipment used to measure the magnetic moment of the sample. The superconducting pickup loop and the low-temperature galvanometer are indicated, with the associated electronics. The driving head assembly is used to modulate the position of the sample at a rate of $2 \mathrm{~s}^{-1}$. the chosen coil geometry the current generated in the flux transformer is linearly proportional to the position of the sample over a range of several $\mathrm{mm}$.

The current in the flux transformer is measured with a flux-gated galvanometer of similar design as reported by Poerschke and Wollenberger. ${ }^{16,19}$ It consists of two high-permeability ring cores which are driven well into saturation with $180^{\circ}$ phase difference. The second harmonic content of the total induction voltage is proportional to the offset caused by the current in the flux transformer. The low-

frequency modulation at the output of the galvanometer is detected with common lock-in techniques.

The static part of the signal, mainly due to the uncompensated flux of the applied magnetic field, can be kept within reasonable limits by occasional heating of part of the flux transformer above its critical temperature which will cause the current to vanish, and by a feedback scheme which couples back to the galvanometer.

The output voltage of the low-frequency phasesensitive detector is linearly proportional to the magnetic moment of the sample for moments up to $0.5 \times 10^{-3} \mathrm{~J} / \mathrm{T}{ }^{20}$ The noise corresponds with $\Delta m=3 \times 10^{-9} \mathrm{~J} / \mathrm{T}\left(3 \times 10^{-6} \mathrm{emu}\right)$ in fields up to $8 \mathrm{~T}$, provided that the magnet is operated in its persistent mode. The instrument has been calibrated against the saturation magnetization of a small nickel sample. The calibration is field dependent and does depend slightly on the magnetic history of the solenoid. The calibration factor is reasonably reproducible when measurements are taken with increasing magnetic 
field. Then, this factor increases approximately with the square of the magnetic field $B$ and has increased by $10 \%$ at $8 \mathrm{~T}$. Even for weak magnetic samples, the corresponding uncertainty in the calibration of the order of a few percent will limit the accuracy of data points above $5 \mathrm{~T}$. At fields of $2 \mathrm{~T}$ and below, the calibration is accurate to approximately $1 \%$. We are not yet certain about the reason for the observed field dependence of the calibration factor.

A continuous flow of helium is maintained in the sample space through a temperature regulated heat exchanger. In this way the sample temperature can be varied between 2.5 and $300 \mathrm{~K}$. Below $100 \mathrm{~K}$ the temperature is determined from the resistance of a carbon resistance thermometer fixed to the inner wall of the cryostat, close to the sample. The resistance thermometer is an epoxy-resin-potted slice of an Allen-Bradley $220-\Omega$ resistor which has been calibrated in situ against a factory-calibrated germanium thermometer; the precision of the temperature determination is better than $1 \%$. Above $100 \mathrm{~K}$ the temperature was determined with a $\mathrm{Au}(0.03$ at. $\% \mathrm{Fe})$ chromel thermocouple linked to the temperatureregulated diffusor. At these temperatures, flowdependent thermal gradients cause an uncertainty of the sample temperature up to several degrees.

\section{RESULTS AND DISCUSSION}

We have measured the magnetization as a function of temperature and magnetic field of samples from a total of 14 sample batches. Only in very few samples the magnetization measured at $4.2 \mathrm{~K}$ was noticeably nonlinear with the magnetic field in a way resembling the results reported earlier. ${ }^{11}$ An apparent temperature dependence of the magnetization has limited the sensitivity for the earliest samples. This apparent temperature dependence is probably due to minor changes in the position of the sample cup and parts of the sample support rod relative to the pickup coils, as a result of the dependence of the length of the sample support rod on the temperature variation over the length of the sample space. Therefore, we have fixed the suspension of indium particles in paraffin directly to the tip of a thin quartz extension of the sample support rod. Then only the sample is within the range of sensitivity of the pickup coils; the contribution to the signal from the quartz extension (in the order of $5 \times 10^{-9} \mathrm{~J} / \mathrm{T}$ ) can be neglected. Because the sample is close to the center of symmetry of the pickup coils, changes in the length of the support rod will have only second-order effects.

For the high fields used in the present experiment it is expected that saturation effects are significant. Therefore care has been taken to include the field dependence of the magnetization in an appropriate way. The data collected as a function of temperature for fixed values of the magnetic field have been successfully interpreted with the following simple model. The ground state of a level characterized by a spin quantum number $S$ will be split into a $(2 S+1)$-fold multiplet when a linear Zeeman term is taken into account. The magnetization can then be calculated in the usual way, and the following expression is obtained for the induced magnetic moment of the sample

$$
m(B, T)-m(B, T \rightarrow \infty)=N g \mu_{B} \frac{\sum_{s}^{+S} m_{s} e^{g m_{s} \mu_{B} B / k T}}{\sum_{m_{s}=-S}^{+S} e^{g m_{s} \mu_{B} B / k T}}
$$

Here $m$ is the magnetic moment of the sample, $N$ is the number of moments with spin quantum number $S$ contained in the sample, for the gyromagnetic ratio $g$ the free electron value has been taken, $\mu_{B}$ is the Bohr magneton, and $k$ is the Boltzmann constant. In Fig. 2 the results are presented for one of the samples. The data of five series of measurements at different values of the magnetic field have been combined and plotted versus $B / T$ for sample 7 . For each series of data collected at a fixed value of the magnetic field, the scaling factor $N$ and the diamagnetic background $m(B, T \rightarrow \infty)$ was adjusted to obtain a best fit. The asymptotic values $m(B, T \rightarrow \infty)$ have been recalculated subsequently, using the average value of the scaling factor $N$ found from the rootmean-square fits to the five sets of data, and have been subtracted from the data. A best fit to the solid line given by Eq. (1) is obtained for $S=1$. However, it should be noted that only for high values of $B / T$ the deviation from linearity is sufficient to allow a clear determination of the value of $S$. Similar results for the temperature-dependent contribution to the magnetization have been obtained for the samples 6 and 8 . For the other samples, the data did not allow such extensive analysis. In Table I the value of $\chi_{m} T$ is given; $\chi_{m}$ is the mass susceptibility, $\chi_{m} T$ is proportional to the high-temperature slope of the curve shown in Fig. 2, and is also proportional to the scaling factor $N$.

For most samples the magnetic moment has been measured as a function of the magnetic field. In Fig. 3 the data are presented for sample 7. A small bend towards positive magnetic moments at fields below $0.4 \mathrm{~T}$ is clearly present; these curves resemble closely the data presented earlier by Meier and Wyder. ${ }^{11}$ The curves show also a deviation from linearity at high magnetic fields, this curvature has been found in all our measurements and is a consequence of the field dependence of the calibration factor of the detection system as noted in the previous section. The measured field and temperature dependence of 


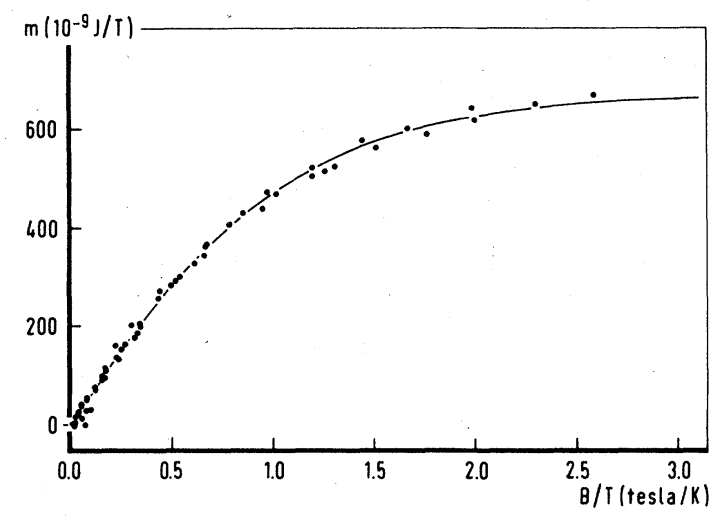

FIG. 2. The temperature dependence of the magnetic moment measured for sample 7 at five different values of the applied magnetic field. The solid curve corresponds to a Langevin function characterized by a spin quantum number $S=1$.

the magnetic moment shown in Fig. 3 is supposed to be built up from the following contributions:

(a) A field dependence of the calibration of the measuring equipment which is quadratic with the magnetic field $\left(1-\alpha B^{2}\right)$.

(b) A temperature-dependent contribution given by Eq. (1) with $S=1$. In some cases the magnitude of this term can be determined from fixed field measurements.

(c) A contribution linear in $B$, associated with the diamagnetic susceptibility of all the atoms in the sample. This contribution is essentially temperature independent.

(d) Finally, in some of the samples, a contribution is seen which is highly nonlinear with the magnetic field for low values of $B$.

Using a root-mean-square minimization procedure, we have adjusted the parameter $\alpha$ so that after sub-

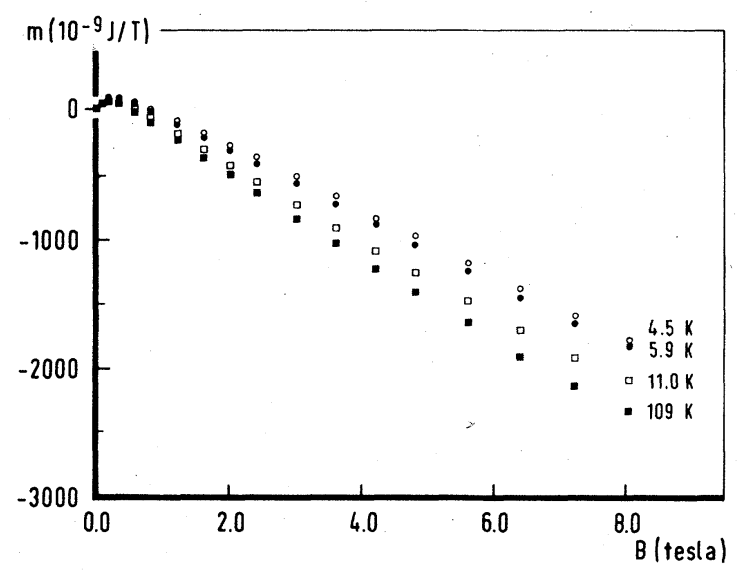

FIG. 3. The field dependence of the magnetic moment, measured for sample 7 at four different temperatures; $0,4.5$ $\mathrm{K} ; \bullet, 5.9 \mathrm{~K} ; \square, 11.0 \mathrm{~K}$; and $\mathbf{m}, 109 \mathrm{~K}$. traction of the temperature-dependent term (b) the data become linear in $B$ for magnetic fields above 1 T. The averaged value of $\alpha$ obtained from ten measured curves $m(B)$ is $\alpha=0.0018 \pm 5 \%$. $\alpha$ has been fixed to this value for all our calculations. The data as shown in Fig. 3 were rescaled with the factor $1 /\left(1-\alpha B^{2}\right)$ to eliminate the field dependence of the calibration. Then, both the temperature-dependent contribution (b), with parameters as deduced from Fig. 2, and a contribution (c), linear with $B$, were subtracted. The value of the slope of this latter term needed to make the contribution (d) constant for high magnetic fields, varied by $2 \%$ only; this variation is considered to be insignificant. The term (d), nonlinear with the magnetic field, subtracted in this way, is shown in Fig. 4(a). This term is virtually tempera-
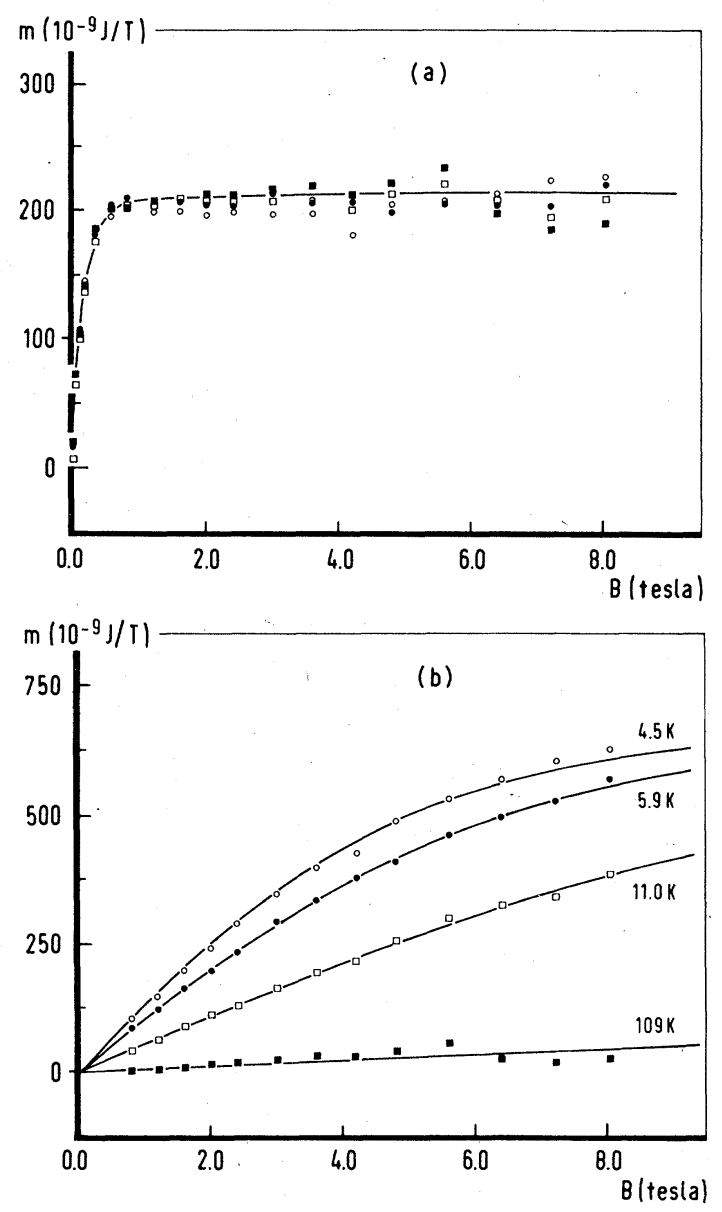

FIG. 4. Different contributions to the magnetic moment, calculated for sample 7. $0,4.5 \mathrm{~K} ; \bullet, 5.9 \mathrm{~K} ; \square, 11.0 \mathrm{~K}$; and a, $109 \mathrm{~K}$. (a) A term highly nonlinear with $B$. This contribution is essentially temperature independent and practically constant for magnetic fields over $1 \mathrm{~T}$. (b) The temperature-dependent contribution to the total magnetic moment obtained after subtraction from the data of a constant level, corresponding to (a), and a term linear with $B$. 
ture independent up to room temperature. In Fig. 4(b) the result is given, obtained after subtraction from the data of a constant level at high magnetic fields, corresponding to contribution (d), and the term linear with $B$. It is obvious that for these measurements significant information on the magnitude of this temperature-dependent term is contained only in the curvature of the low-temperature curves when a spread of several percent in the slope of the linear term is still insignificant. For some samples the value of $\chi_{m} T$ could be inferred roughly using this procedure. The experimental results of this analysis are collected in Table I for a number of samples and for some blank paraffin specimens.

From this table it can be seen that the values of the mass susceptibility found for a number of samples and measured on five blank paraffin samples, B1 1 through $\mathrm{Bl} 5$, are overlapping. All blank samples have been prepared differently. Some relevant aspects of the preparation procedure are summarized in Table II. B1 2 was taken directly from the paraffin supply and pressed into a cylindrical shape. In three different runs, paraffin has been exposed to all the production steps, except the evaporation of indium; B1 3 and B1 4 have been prepared from the same batch. The samples B1 3 and B1 5 have been prepared, as B1 2, by pressing the paraffin into shape under air. To avoid the inclusion of cavities containing air, we have melted alternatively some paraffin in a cylindrical holder: this procedure has been used with $\mathrm{B} 11, \mathrm{~B} 14$, In 6 , and In 7. Sample In 8, prepared from the same batch as In 7 , has been pressed into shape.

The value found for the susceptibility $\chi_{m}$ does correlate with the sample preparation technique. The susceptibility found is probably due to atmospheric oxygen, trapped in the paraffin matrix; also the value $S=1$ found from the temperature and field dependence of the susceptibility is consistent with the presence of oxygen.
The nonlinear contribution to the magnetic moment of the type shown in Fig. 4(a) resembles the magnetization curve of a ferromagnet. A significant contribution of this type has been found in four samples and in one blank out of a total of seventeen samples and five blanks. Moreover, the effect showed up in three samples and one blank which have been measured consecutively. It is most probably due to a contamination with small pieces of iron. The saturation magnetic moment of $210 \times 10^{-9} \mathrm{~J} / \mathrm{T}$ for sample 7 corresponds to a total of $1 \mu \mathrm{g}$ iron, a 20 ppm contamination of the sample.

So far we have only considered the significance of the experimental results. In the following we will estimate the signal expected from quantum-size-effect predictions. The most pronounced effect in the electronic susceptibility of small metallic particles is the susceptibility of the unpaired spin in odd particles. The Pauli spin paramagnetism dominates at high temperature, because the number of partially filled states is increased by the thermal spreading. For bulk metals the Pauli spin paramagnetism is therefore temperature independent; for very small crystallites at temperatures below $\delta / k$, the Pauli spin paramagnetism will vanish as a result of the freezing out of the neighboring levels. The quantum size parameter $\delta$ can be estimated to be ${ }^{1}$

$$
\delta=\frac{4}{3} \epsilon_{F} / N
$$

The Pauli susceptibility is given by ${ }^{21}$

$$
\chi_{\text {Pauli }}=\mu_{0} \mu_{B}^{2} \rho\left(\epsilon_{F}\right)=1.9 \times 10^{-5}
$$

for indium. The volume susceptibility associated with one unpaired spin in a particle of volume $V_{0}$ is

$$
\chi=\mu_{0} \mu_{B}^{2} / k T V_{0}
$$

When the quantum size parameter scales with $N^{-1}$,

\begin{tabular}{|c|c|c|c|c|c|}
\hline & \multirow{3}{*}{$\begin{array}{l}\text { Helium } \\
\text { pressure }\end{array}$} & \multicolumn{2}{|c|}{ Preparation of the sample batch } & \multirow{2}{*}{\multicolumn{2}{|c|}{ Preparation of the sample }} \\
\hline & & \multirow{2}{*}{$\begin{array}{l}\text { Production } \\
\text { time }\end{array}$} & \multirow{2}{*}{$\begin{array}{c}\text { Oven } \\
\text { temperature }\end{array}$} & & \\
\hline & & & & Pressed & Melted \\
\hline B। 1 & 10 Torr & $48 \mathrm{~h}$ & . . & & $\mathrm{X}$ \\
\hline B| 2 & & rectly from th & in supply & $\mathrm{X}$ & \\
\hline B| 3 & 10 Torr & $29 \mathrm{~h}$ & $\cdots$ & $\mathrm{X}$ & \\
\hline B) 4 & idem & idem & $\cdots$ & & $\mathrm{X}$ \\
\hline B1 5 & 10 Torr & $44 \mathrm{~h}$ & $\cdots$ & $\mathrm{X}$ & \\
\hline In 6 & 10 Torr & $31 \mathrm{~h}$ & $1195 \mathrm{~K}$ & & $\mathrm{X}$ \\
\hline In 7 & 10 Torr & $48 \mathrm{~h}$ & $1175 \mathrm{~K}$ & & $\mathrm{X}$ \\
\hline In 8 & idem & idem & idem & $\mathrm{X}$ & \\
\hline
\end{tabular}
and hence with $V_{0}^{-1}$ [as in Eq. (2)], one obtains the

TABLE II. Preparation characteristics of the samples. 
following relation for this regime

$$
\chi \simeq 6.8 \times 10^{-6} \delta / k T .
$$

From Eq. (5) it is clear that the susceptibility due to the one unpaired spin has become comparable to the Pauli susceptibility when the latter starts to deviate from the normal bulk value. ${ }^{1,3}$ The paramagnetic susceptibility will dominate when $V_{0} T<4.2 \times 10^{-25}$ $\mathrm{m}^{3} \mathrm{~K}$. For $T=4.2 \mathrm{~K}$, this leads to a particle diameter smaller than $6 \mathrm{~nm}$. The magnetic moments induced in a sample with typical characteristics as found in Table I and calculated using Eqs. (3) and (4) are almost an order of magnitude below the resolution of the present magnetometer. The value of $\chi_{m} T$ calculated is three orders of magnitude smaller than the residual susceptibility found for the paraffin matrix. Therefore, the susceptibility of the unpaired spin in odd indium particles cannot have played a role in the present investigation. To account for the low saturation field observed in the magnetization of small indium particles embedded in paraffin a model has been proposed $^{11,12}$ where instead of spin magnetic moments the moments associated with high angular momentum ( $L=13$ ) are considered. Then, the expected susceptibility will be orders of magnitude higher, but still does not exceed the residual susceptibility of $7 \times 10^{-9}(1 / T) \mathrm{m}^{3} / \mathrm{kg}$. Therefore neither of the models proposed can explain the present results quantitatively.

We did not cover the same temperature interval, but the temperature-dependent contribution shown in Fig. 3 can be calculated using Eq. (1) for the values of the magnetic field and temperature reported in Ref. 11. With the parameters of sample 7 we have calculated the magnetic moments expected for 10 , $4.2,3.5,2.5$, and $1.5 \mathrm{~K}$. From these curves we have subtracted, following Meier and Wyder, ${ }^{11}$ a term linear in $B$ found from the fit of a linear function to these curves in the magnetic field range from 2 to 5 T. When subsequently an averaged nonlinear background is added, calculated from the data in Fig. $4(\mathrm{a})$, the apparent temperature dependence of the

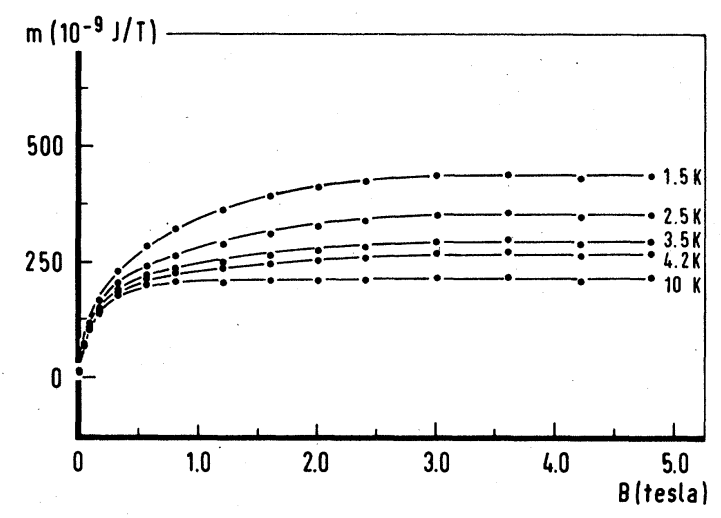

FIG. 5. The magnetic moment of sample 7 at low temperatures calculated using the analysis reported by the authors of Ref. 11. The relative position of the saturation levels at $1.5,2.5,3.5$, and $4.2 \mathrm{~K}$ agrees very well with the results reported (Ref. 11).

magnetic moment is strongly reduced. It can be seen from the results shown in Fig. 5 that the relative position of the "saturation" levels is remarkably well reproduced. However, the temperature-dependent contribution is much bigger relative to the temperature-independent background. In view of the remarkable similarity of our calculated result with the previously reported anomalous magnetization of indium particles, we suggest that those results can be interpreted along the same lines. The temperaturedependent contribution to the magnetization does not reveal a quantum size effect in the small indium particles.

\section{ACKNOWLEDGMENT}

Part of this work has been supported by the Stichting voor Fundamenteel Onderzoek der Materie (FOM) with financial support from the Nederlandse Organisatie voor Zuiver Wetenschappelijk Onderzoek (ZWO).
'R. Kubo, J. Phys. Soc. Jpn. 17, 975 (1962).

${ }^{2}$ H. Fröhlich, Physica (Utrecht) 4 , 406 (1937).

${ }^{3}$ R. Denton, B. Mühlschlegel, and D. J. Scalapino, Phys. Rev. B I, 3589 (1973).

${ }^{4}$ W. D. Knight, R. Monot, E. R. Dietz, and A. R. George, Phys. Rev. Lett. 40, 1324 (1978).

5J. Charvolin, C. Froidevaux, C. Taupin, and J. M. Winter, Solid State Commun. 4, 357 (1966).

${ }^{6} \mathrm{Ph}$. Yee and W. D. Knight, Phys. Rev. B 11, 3261 (1975).

${ }^{7}$ A. Kawabata, J. Phys. Soc. Jpn. 29, 902 (1970).

${ }^{8}$ R. Monot and J.-L. Millet, J. Phys. (Paris) Lett. $\underline{37}$, L45 (1976).
${ }^{9}$ R. F. Marzke, W. S. Glaunsinger, and M. Bayard, Solid State Commun. 18, 1025 (1976).

${ }^{10}$ D. A. Gordon, R. F. Marzke, and W. S. Glaunsinger, J. Phys. (Paris) 38, C2-87 (1977).

${ }^{11} \mathrm{~F}$. Meier and P. Wyder, Phys. Rev. Lett. 30, 181 (1973).

${ }^{12}$ A. P. van Gelder, Phys. Rev. B 10, 2144 (1974).

${ }^{13}$ Indium, purity better than $99.999 \%$, Johnson Matthey Chemical Ltd.; paraffin, solidification range $42-44^{\circ} \mathrm{C}$, Merck 7159, E. Merck, Darmstadt, BRD; and the helium was evaporated directly from the liquid.

${ }^{14}$ C. G. Granqvist and R. A. Buhrman, J. Appl. Phys. 47, 2200 (1976). 
${ }^{15}$ R. R. Gelsing and H. van Kempen, in Proceedings of the 4th International Cryogenic Engineering Conference, Eindhoven, 1972, edited by Transcripta Service, London (IPC Science and Technology, Guildford, Surrey, 1972), p. 233.

${ }^{16} \mathrm{H}$. van Kempen, J. A. A. J. Perenboom, and P. Wyder (unpublished)

${ }^{17}$ S. Foner, Rev. Sci. Instrum. 30, 548 (1959).

${ }^{18} \mathrm{M}$. Springford, J. R. Stockton, and W. R. Wampler, J. Phys. E 4,1036 (1971).
${ }^{19} \mathrm{R}$. Poerschke and $\mathrm{H}$. Wollenberger, Cryogenics $\underline{10}, 333$ (1970).

${ }^{20} \mathrm{We}$ have used mksa units throughout this paper. The conversion from mksa to cgs values is as follows: for the magnetic moment $1 \mathrm{~J} / \mathrm{T}=10^{3} \mathrm{emu}$; for the volume susceptibility $\chi^{\mathrm{mksa}}=4 \pi \chi^{\mathrm{cgs}}$, dimensionless; for the mass susceptibility $\chi_{m}^{\mathrm{mksa}}=4 \pi \times 10^{-3} \chi_{g}^{\mathrm{cgs}}$, in units of $\mathrm{m}^{3} / \mathrm{kg}$.

${ }^{21} \mathrm{C}$. Kittel, Introduction to Solid State Physics, 5th ed. (Wiley, New York, 1976), p. 450. 